\title{
MATEMÁTICAS EN LAS REDES \\ Y CIENCIAS SOCIALES
}

\section{MATHEMATICS IN SOCIAL SCIENCES \\ AND NETWORKS}

\author{
MATEMÁTICA NAS REDES E \\ CIÉNCIAS SOCIAIS
}

SOCORRO MÁRQUEZ _ Félix Oscar

Doctor en Ciencias Administrativas, Docente tiempo completo de la Universidad de la Salle, Email: fsocorro@unisalle.edu.co, Colombia.

Recibido: 10 de junio de 2019

Aprobado: 21 de julio de 2019

DOI: http://dx.doi.org/10.22267/rtend.192001.116

\section{RESUMEN}

Varios autores sostienen que las matemáticas están íntimamente vinculadas con las redes y las ciencias sociales, por lo que su uso, importancia y utilidad no pueden ser obviadas al momento de iniciar una carrera en alguna de las disciplinas asociadas a estas ciencias. En el artículo se explora cómo las matemáticas pueden encontrarse en la cotidianidad y hasta en expresiones culturales donde parecieran no tener cabida, para finalmente concluir que todo profesional debe desarrollar un pensamiento analítico, crítico y matemático independientemente de los estudios que desee completar.

Palabras clave: ciencias sociales, matemáticas, redes

JEL: C65 


\begin{abstract}
Several authors maintain that mathematics is closely linked to social sciences and networks, so its use, importance, and usefulness cannot be ignored when starting a career in one of the disciplines associated with these sciences. The article explores how mathematics can be found in daily life and even in cultural expressions where they seem to have no place, and finally conclude that all professionals must develop an analytical, critical and mathematical thought independently of the studies they wish to complete.
\end{abstract}

Keywords: science, social, mathematics, networks

\title{
JEL: C65
}

\section{RESUMO}

Vários autores argumentam que a matemática está intimamente ligada a redes e ciências sociais, de modo que seu uso, importância e utilidade não podem ser ignorados ao iniciar uma carreira em qualquer uma das disciplinas associadas a essas ciências. $\mathrm{O}$ artigo explora como a matemática pode ser encontrada na vida cotidiana e até mesmo em expressões culturais onde parecem não ter lugar, para finalmente concluir que todo profissional deve desenvolver um pensamento analítico, crítico e matemático, independentemente dos estudos que deseja concluir.

Palavras-Chave: ciências sociais, matemática, redes JEL: C65

\section{INTRODUCCIÓN}

Puede leerse en uno de los libros de Mcintosh-Scott (2018); la frase "hay tres tipos de personas en el mundo, aquellas que pueden contar y aquellas que no"1 (p.177) atribuida a Homero Simpson, el conocido personaje de caricaturas de la cadena estadounidense FOX.

1 La versión en inglés dice: There are three kind of people in this world... Those who can count, and those who can't 
Como puede observarse, la afirmación realizada por el personaje es incorrecta, pues inicia señalando que hay tres tipos de personas, pero sólo describe a dos de ellas.

Lo anterior nos invita a reflexionar, ya que la fuente del aforismo proviene de un personaje de ficción con 30 años de existencia, cuyas situaciones hilarantes parecieran estar destinadas, principalmente, a la diversión y el entretenimiento, no obstante, Homero, ese personaje ficticio, no sólo habla de las matemáticas, sino que hace que los espectadores, luego de escuchar su afirmación, comprendan lo errado de la misma, es simple, si ha dicho 3 y sólo explica 2, falta, evidentemente, 1 tipo de persona o solo hay 2 , lo que se traduce a la simple operación matemática: $3-2=1$.

Pero ¿no se trata de divertir? ¿Por qué recurrir a las matemáticas en una caricatura? Para responder esas dos preguntas es necesario comprender que las caricaturas están íntimamente relacionadas con las Ciencias Sociales.

En un debate formulado y organizado por Ospina (2010) y reseñado por la revista de Estudios Sociales, sobre las caricaturas, puede leerse una afirmación de Catalina Ruiz-Navarro que reza: "la caricatura hace anotaciones constantes sobre la actualidad, sobre todo la política, y como su posición es inmediata, y crítica, construye una historia con las mismas características” (p. 2)

Así mismo Ospina (2010) reseña lo expresado por Alejandro Martín, quien afirma:

Yo no creo que podamos llamar al caricaturista periodista, más bien columnista de opinión. Yo ligo el periodismo a la investigación. Y el caricaturista no hace eso, más bien opina sobre lo que está sucediendo, sobre lo que todo el mundo conoce. (Ospina, 2010, p. 3)

Y es así que, en ambas afirmaciones, las ciencias sociales están presentes, en una a través de la posición crítica y en la otra a través de la comunicación de posturas y opiniones.

Se infiere, entonces, que el aforismo de Homero Simpson, citado por McintoshScott (2018), no representa ni una postura ni una opinión, pero sí puede ser considerado como una crítica a la carencia de educación formal sobre el tema, la 
misma que el caricaturista representa en la figura del personaje que construye, lo que respondería por qué están presente las matemáticas en el dibujo animado, ya que, de manera preliminar, pareciera que se intenta destacar el desconocimiento del personaje con relación al tema.

En lo que respecta a si la caricatura trata de divertir o no, parece correcto suponer que, ante tal afirmación, a una persona que haya realizado el cálculo matemático antes descrito, pudiese parecerle gracioso por su inexactitud matemática, o, a la vez, podría causarle gracia debido al retrato de la carencia de formación básica que en ella se expresa, a modo de sátira.

Es así, entonces que se destaca, de manera indirecta, la importancia del conocimiento matemático en una trama que pareciera no requerir de ello $\mathrm{y}$, a su vez, invita a reflexionar sobre su presencia en escenarios distintos a los que, tradicionalmente, están asociados a las matemáticas.

Si se dudara de la inclusión de las matemáticas en series animadas de televisión, donde pareciera que las matemáticas no tienen cabida; cabe señalar que existe un libro titulado Los Simpson y las Matemáticas, donde Singh (2016), el autor del mismo, afirma, en el capítulo 0 que "los Simpson están profundamente enamorados de los números, y su deseo fundamental es inyectar fragmentos de matemáticas en el subconsciente de los espectadores" (p. 5)

Con base en lo anterior surgen dos preguntas ¿cuál es el papel de las matemáticas en las redes y ciencias sociales? ¿podría un profesional de las ciencias sociales obviar el uso y utilidad de las matemáticas en su ejercicio?

Este artículo pretende dar respuesta a esas preguntas, apoyándose en autores especialistas en estos temas y otras fuentes de información disponibles, para identificar y extraer los elementos que faciliten describir, documentar y sustentar la importancia del conocimiento y uso de las matemáticas en las ciencias sociales, aun cuando de manera directa esos autores o escritos no hayan enfocado sus estudios $\mathrm{y} / \mathrm{o}$ propuestas a tal fin.

Lo anterior se hace desde una perspectiva académica, aportando con ello información, como resultado del análisis e investigación, dirigida a quienes estudian las ciencias sociales para evitar el contacto con las matemáticas. 
El contenido de este artículo se limita a comentar, señalar y argumentar, con base en la experiencia empírica y el contraste documental los aspectos que, desde la perspectiva del autor, destacan la importancia de las matemáticas en las ciencias sociales.

Por lo tanto, la metodología utilizada es de tipo cualitativa, con énfasis en la investigación documental. Los contenidos recopilados provienen de libros, artículos, videos, revistas, publicaciones periódicas y otras fuentes que, a juicio del investigador, ofrecen el contenido que se desea relacionar y/o vincular al propósito de la investigación.

\section{LAS MATEMÁTICAS, COTIDIANIDAD Y REDES SOCIALES}

Se atribuye a Galileo Galilei (1564-1642) la frase "las matemáticas son el alfabeto con el que Dios escribió el universo", cuyo contenido y profundidad invita a suponer que no hay nada en el universo que no esté vinculado a las matemáticas.

Y, de ser cierto lo expresado en el párrafo anterior, ninguna ciencia, estudio, propuesta, conclusión o pensamiento escapa del razonamiento matemático, por más simple que sea, tal y como quedó demostrado con la afirmación realizada por el personaje de los Simpsons.

Un ejemplo de las matemáticas, en contextos no académicos ni laborales, puede encontrarse en las redes sociales, herramientas éstas que también son usadas para estudios, proyecciones y alcance de noticias, propuestas, productos y servicios en diferentes áreas, tal y como lo explica Celaya (2008), citado por Herrera (2012), "las redes sociales son lugares en Internet donde las personas publican y comparten todo tipo de información, personal y profesional, con terceras personas, conocidos y absolutos desconocidos", (Herrera, 2012, p. 123)

Si bien Socorro (2004) no reduce las redes sociales al ambiente virtual, como lo hace Celaya (2008) citado por Herrera (2012), el uso de los dispositivos celulares ha orientado su ubicación primordialmente en ese escenario.

Ahora bien, puede inferirse, entonces, que, si la información que se comparte es de tipo profesional, debe estar sustentada y parametrizada de acuerdo a la profesión y es precisamente las matemáticas la que permiten hacerlo. 
Para sustentar la afirmación anterior se puede hacer uso de lo expresado por Salgado (2010) quien plantea que las matemáticas "suelen ser caracterizada como la ciencia que se encarga de las cantidades y formas, sus propiedades y relaciones valiéndose de la utilización de números y símbolos” (p.4).

Si se relaciona lo explicado por Salgado (2010) con una red social como Instagram, por ejemplo, pueden extraerse los elementos presentes en su conceptualización dentro del uso de la mencionada red; en ella se registra el número de personas que siguen la cuenta y el numero de personas a las que la cuenta sigue, así como el número de publicaciones realizadas y la cantidad de likes recibidos, o sea, se pueden observar en cantidades las veces que se interactúa con su contenido y, adicionalmente, las relaciones entre ellos y la cuenta que se posee, expresados estos en "números y símbolos".

Lo anterior muestra, de manera simple, como en una actividad ordinaria, de uso cotidiano, las matemáticas se encuentran presente.

Aunque pudiese considerarse como algo obvio, existe la creencia popular de requerirse menos el uso de las matemáticas en disciplinas sociales comparadas con las ciencias puras, cuando en realidad, las matemáticas están presentes en todo lo que se hace.

Adicionalmente, Salgado (2010) afirma también que los elementos que conforman las matemáticas son "lógica e intuición, análisis y construcción, generalidad y particularidad" (Ídem), elementos estos que pueden ser rastreados en actividades cotidianas y comunes de la sociedad y por ende pueden alimentar y facilitar el empleo de la ciencia en ello.

Al retomar el ejemplo de las redes sociales, encontramos que en Twitter es posible conocer el comportamiento de un trino, ello conlleva al análisis e impacto del mismo y, dependiendo de donde se encuentre, ya sea una publicación general o en una lista, se puede hacer dicho análisis de manera general o particular, lo que coincide perfectamente con lo mencionado por Salgado (2010). 
Ahora bien, de acuerdo al IPEBA² (2013), la matemática “desarrolla en el estudiante competencias que le permitan plantear y resolver con actitud analítica los problemas de su contexto y de la realidad, de manera que pueda usar esas competencias matemáticas con flexibilidad en distintas situaciones" (p. 7). Si bien es cierto que lo expuesto por IPEBA (2013) aplica no sólo para los estudiantes, no es menos cierto que el rechazo al estudio de las matemáticas se observa más en esa población.

Según Garzón (2013), citado por Caballero y Espínola (2016):

pese a la importancia de las matemáticas hay un fuerte rechazo por parte de los estudiantes para su aprendizaje, ya que la perciben como aburrida, acartonada, compleja, complicada, difícil de entender, (...) la gran mayoría de los jóvenes odian las matemáticas (Caballero y Espínola, 2016, p. 149)

Caballero y Espínola (2016) continúan diciendo que:

En este rechazo influyen las características propias de las matemáticas como ser precisa; es decir, no ambigua, que es abstracta. Además del estereotipo transmitido con frecuencia por padres, amigos y familiares que comentan sus experiencias no gratas en esta área del conocimiento. Por tanto, la misma sociedad se ha encargado de promover que las matemáticas son difíciles y destinadas para los “más inteligentes” (Caballero y Espínola, 2016, ídem)

Puede decirse, entonces, por lo antes expuesto, que si las matemáticas están en todo lo que hacemos no podrían circunscribirse únicamente a las personas más inteligentes, pues, de lo contrario, las redes sociales no formarían parte de la expresión contemporánea como hoy en día lo hacen. Y si fuesen aburridas y acartonadas no aparecerían en expresiones sociales como cortos animados o caricaturas.

Así mismo, para Caballero y Espínola (2016), "los estereotipos o prejuicios en muchos casos se arrastran de generación en generación, mismos que en ocasiones llegan a bloquear la mente de los niños y jóvenes". (Ídem), lo que se traduce en un reto para quienes deben enseñar y destacar la importancia de las matemáticas

2 Instituto peruano de evaluación y certificación de la calidad de la educación básica 
en todos los campos del saber, demostrando, de manera simple o significativa, cómo las matemáticas influyen en nuestros actos, pensamientos y decisiones, tal y como lo afirma Alsina (2014) ${ }^{3}$.

\section{LAS MATEMATICAS Y LAS CIENCIAS SOCIALES}

Así como las matemáticas se encuentran presentes en las caricaturas, cortos animados, redes sociales y, finalmente, en la cotidianidad, las podemos encontrar en las ciencias sociales como la base donde se sustentan sus aportes.

Para Prats (s.f) existen dos tipos de ciencias:

[las] formales (lógica y matemáticas) que crean su propio objeto de estudio con una vaga referencia a realidad con la que se relacionan mediante el lenguaje simbólico. Y fácticas que tratan de explicar el mundo de la naturaleza física o sociocultural (Prats, s.f., p.5)

No obstante, a pesar de esta división, no puede afirmarse que las matemáticas están ausentes en alguna de ellas, pues para explicar al mundo se requiere lógica y cálculo, o al menos eso puede entenderse de lo expresado por Bernis (2008) cuando afirma que "la metodología científica (...) no participa del atractivo de las especulaciones a rienda suelta, de la esperanza de las panaceas y del sobresalto producido por teorías omnicomprensivas" (p. 31)

Para comprender la relación entre las ciencias sociales y las matemáticas se hace necesario conocer primero qué debemos entender por las primeras.

Prats (s.f) afirma que "las ciencias sociales son una unidad cimentada en la diversidad" (p. 7), para luego señalar que "nos atrevemos a hablar de ciencias sociales para el estudio de lo social y no de una (única y exclusiva) ciencia social: una panciencia social" (p. 8)

Por su parte Manzanos (2002) afirma que "cada una de las disciplinas de las ciencias humanas y sociales (...) trata de configurar, en torno a sus ámbitos de definición, una cosmovisión particular del mundo social" (p.16).

3 Véase: https://www.educaixa.com/es/modal-recurso?resourcePK=14114512\&isBanner=true 
Manzano (2002) lista entre las disciplinas propias de las ciencias sociales a la economía, el derecho, la psicología, la estadística, la comunicación, la lingüística y el urbanismo.

De las disciplinas listadas por Manzano (2002), se destacan la economía y la estadística por su directa relación con las matemáticas.

Explica González (s.f) que:

En la economía, contribuyeron en gran medida todas las corrientes de pensamiento: las inductivas, las deductivas, las historicistas, las institucionalistas, las estructuralistas, las cuantitativistas y las matemáticas. Sobre todo, estas últimas, ya que las matemáticas se han aplicado con carácter general en todas las ciencias, hasta tal punto que una teoría no asentada en fundamentos matemáticos no parece científica, sino especulativa (González, s.f., p. 486)

Por su parte, la Organización para la Cooperación y el Desarrollo Económicos, citado por IPEBA (2013) afirma que:

los aprendizajes que se logran a partir de la Estadística y el cálculo de probabilidades deben adquirir hoy mayor importancia de la que tenían en el pasado, pues se han constituido en herramientas que ayudan al estudiante a organizar y profundizar su conocimiento sobre la realidad que lo circunda (IPEBA, 2013, p. 8)

Es por ello que, hablar de economía y estadística, es hablar de las matemáticas, pero ¿qué ocurre con el resto de las disciplinas? ¿también tienen relación con las matemáticas?

Afirma Salgado (2010) que "las matemáticas son útiles al abogado porque le permiten realizar cálculos que el estudio, interpretación y aplicación del derecho exigen" (p. 1), por lo que el estudio de las leyes no está divorciado del uso del cálculo y las proyecciones matemáticas.

Para Bernis (2008) “la utilización del método matemático en Psicología constituye un poderoso instrumento analítico -de lo que debe ser consciente el psicólogoque debe aplicarse en todos aquellos casos en que resulte conveniente" (p. 21), 
lo que se entiende, desde la perspectiva del autor, que dicha conveniencia surgirá para soportar decisiones clínicas, organizacionales o particulares surgidas del uso profesional de la psicología.

En lo que respecta a comunicación y lingüística, Fernández (2011) explica que:

la característica de la comunicación humana induce a la conjetura de la presencia de un sistema lingüístico dual, específicamente el lenguaje matemático, ya sea aritmético, algebraico, geométrico o lógico. Quizá por ello, la matemática más que números y operaciones básicas elementales, es también, definiciones o conceptos y procesos de pensamiento como, por ejemplo: tamaños, medidas, semejanzas y diferencias, clasificación, seriación, análisis, síntesis, entre otros, los cuales son necesarios y fundamentales para que se consolide en el sujeto las dimensiones de las capacidades humanas. (Fernández, 2011, p. 2)

Lo anterior conduce a comprender la estrecha relación que existe entre la expresión del lenguaje y su conexión con las matemáticas, ya que, por deducción, una cosa no podría existir sin la otra.

En cuanto al uso y conocimiento de las matemáticas en los medios de comunicación, aunque no fueron listados anteriormente, pero presentes en el quehacer comunicativo humano, explica Jiménez (2018) que: Quizás una parte de las matemáticas que más se usa en los Medios de Comunicación sea la Estadística (...), continuando con una cantidad de números y/o porcentajes, seguidos de tablas y gráficos estadísticos, para que el lector entienda más rápido lo que lee (...). Las estadísticas que aparecen en la televisión a lo largo de un partido de fútbol, baloncesto, estadísticas (de muertos en carretera durante el fin de semana, son cosa habitual y tienen en común que son un conjunto de datos numéricos sobre una cuestión presentada en tablas o gráficos. (Jiménez, 2018, p.1)

Por todo lo anterior puede decirse que no habría ciencias sociales sin la participación y sustento de las matemáticas, o al menos no de una manera científicamente aceptada, ya que el uso de las matemáticas le otorga la rigurosidad que la ciencia exige y permite el avance y desarrollo de las disciplinas asociadas a ellas. 


\section{CONCLUSIONES}

Si bien es cierto que en la introducción se habló de una frase de un personaje ficticio, como lo es Homero Simpson, donde, de manera reflexiva se observa un desconocimiento básico de las matemáticas y que con ello se pretendió inducir un ejercicio matemático simple para ilustrar la presencia del cálculo y las ecuaciones en escenarios donde pareciera que no deberían estar; no es menos cierto que dicha referencia buscaba, además de lo comentado, destacar que nada es ajeno a las matemáticas y con ello dar respuesta, con base en los aportes de distintos autores a las preguntas que, como parte del proceso investigativo, se realizaron en el mismo apartado.

Con relación al papel de las matemáticas en las redes y ciencias sociales, parece correcto afirmar, en lo que respecta a las redes, que las matemáticas están presentes en el enjambre comunicacional en las que ellas se desarrollan, observándolas desde los seguidores y likes hasta la influencia e impacto de las interacciones.

Podría ser una decisión, para quienes usan las redes sociales, quedarse únicamente con la información, sin ir más allá y ejercer la lógica deductiva y el análisis para incrementar su participación en las mismas, pero de ser así ¿qué caso tendría?

Las matemáticas impulsan la comunicación y facilitan la comprensión en el mensaje y su relación con el ambiente donde éste se esparce, por lo que su papel en las redes sociales es fundamental e inevitable.

Ahora bien, en cuanto al papel de las matemáticas en las ciencias sociales, puede decirse que el mismo funge de sustento y le otorga rigurosidad científica, a pesar de lo que expresa Suárez-Iñiquez (2004) cuando afirma que las "ciencias sociales no pueden tener el mismo grado de exactitud que las ciencias naturales [pero] sí pueden aplicar las herramientas que proporciona la ciencia en sus análisis” (p. 25).

Ahora bien, con relación a si un profesional de las ciencias sociales podría obviar el uso y utilidad de las matemáticas en su ejercicio, puede decirse, con base en lo investigado, que no podría considerarse un ejercicio profesional per se si las matemáticas no se encuentran involucradas en él, por lo que parece lógico afirmar que la respuesta a esa pregunta es: no, no puede. 
El argumento principal de esa respuesta subyace en la afirmación antes mencionada de Galileo Galilei, es simple, si las matemáticas son el alfabeto con el que Dios escribió el universo, es imposible suponer que existan ramas del saber y las ciencias que no requieran de ella para sustentarse.

No obstante, parece prudente reconocer que no todas las personas se sienten interesadas por el uso del pensamiento matemático y lógico, ya sea, como se comentó, por las experiencias transmitidas por sus familiares y amigos, o por sus propias experiencias y, en otros casos, simplemente por no considerarlas importantes para sí, aunque deban hacer uso de las matemáticas en operaciones simples o sutilmente complejas.

Lo anterior se comprendería en aquellos casos en los que optar por un título profesional no es una meta, pero quedaría completamente descartado en los casos donde se quiera ejercer una profesión, pues, como ya se comentó, las matemáticas forman parte tanto de las ciencias puras como de las ciencias sociales.

Más allá de todo ello, debe comprenderse que en el quehacer profesional el uso de las matemáticas definirá el futuro de quienes culminen una carrera, tal y como lo sugiere Andreas Schleicher, citado por Caballero y Espínola (2016); quien fuere coordinador de PISA, al afirmar que, "la educación que reciba hoy, será su economía mañana” (p. 150), y, como se explicó, la economía y las matemáticas van de la mano.

\section{RECOMENDACIONES}

Si usted está estudiando alguna carrera asociada a las ciencias sociales tenga presente las siguientes recomendaciones:

- Dedique tiempo al conocimiento de las matemáticas y su aplicación en su carrera, ello le otorgará una ventaja competitiva y comparativa frente a quienes no lo hacen.

- Si no entiende una ecuación matemática de uso común en su rama de estudios no la obvie, por el contrario, asesórese y no descanse hasta comprenderla, tómelo como un reto personal, entenderla, usarla y explicarla validará su condición de profesional. 
- Si quiere ser reconocido como profesional debe manejar las matemáticas de su área, eso evitará que sus opiniones y propuestas sean consideradas especulaciones.

- Busque apoyo en personas dedicadas a las matemáticas para soportar sus ideas y proposiciones, eso le permitirá poseer una visión analítica más amplia y facilitará visualizar escenarios que, tal vez, haya omitido.

- No vea las matemáticas como su enemiga, es una aliada, una herramienta, un sustento.

- No base su concepción de las matemáticas en opiniones de terceros, experiméntela de primera mano.

- Busque en las matemáticas respuestas prácticas a sus problemas, expectativas y requerimiento, así podrá experimentar su utilidad.

\section{REFERENCIAS}

(1) Alsina, C. (2014). Matemáticas y la vida cotidiana. Las matemáticas a nuestro servicio. Palma de Mallorca, España: Fundación bancaria caixa d'estalvisi .

(2) Bernis, J. M. (2008). El estudio operativo de la psicología, una aproximación matemática. Tortosa: Universidad Nacional de Educación a Distancia (España). Centro Asociado de Tortosa.

(3) Caballero Jiménez, F., \& Espínola Reyna, J. G. ( 2016). El rechazo al aprendizaje de las matemáticas a causa de la violencia en el bachillerato tecnológico. Ra Ximhai, 143-161.

(4) Fernández, A. R. (10 de junio de 2011). ilustrados.com. Recuperado el 2019, de El Lenguaje y la Matemática. Un binomio fundamental para su enseñanza y aprendizaje: http://www. ilustrados.com/tema/7385/Lenguaje-Matematica-binomio-fundamental-para-ensenanza. html

(5) González, E. E. (s.f.). personal.us.es. Recuperado el 10 de junio de 2019, de Historia del Pensamiento Ecomómico. Tema 30. La economía matemática: personal.us.es/escartin/ Economia_Matematica.pdf

(6) Herrera, H. H. (2012). Las redes sociales: una nueva herramienta de difusión. Reflexiones, 121-128. 
(7) IPEBA. (2013). Mapas de Progreso del Aprendizaje: Matemática, Estadística y probabilidad. Lima: Ministerio de Educación.

(8) Jiménez, E. B. (2018). smpm.es. Recuperado el 12 de junio de 2019, de Matemáticas y medios de comunicación: https://www.smpm.es/component/content/article/39-dia-escolar/ matematicas-y-prensa/67-matematicas-y-medios-de-comunicacion

(9) Manzanos Bilbao, C. (2002). Las ciencias sociales: convergencias disciplinarias y conocimiento de fronteras. El caso de la sociologia. Revista Mexicana de Ciencias Políticas y Sociales, 13-65.

(10) Mcintosh-Scott, A. (2018). Key Concepts In Nursing And Healthcare Research Sage Key Concepts Series. Londres: SAGE.

(11) Ospina, L. (2010). Sobre la caricatura. Revista de Estudios Sociales. Recuperado el 17 de junio de 2019, de http://www.scielo.org.co/scielo.php?script=sci_arttext\&pi$\mathrm{d}=\mathrm{S} 0123-885 \mathrm{X} 2010000100012$

(12) Prats, J. (s.f.). ub.edu. Recuperado el 10 de junio de 2019, de Las ciencias sociales en el contexto del conocimiento científico: http://www.ub.edu/histodidactica/images/documentos/pdf/prats-\%20que\%20son\%20las\%20ccss.pdf

(13) Salgado, D. C. (2010). Matemáticas aplicadas al derecho. México: Porrúa.

(14) Singh, S. (2016). Los Simpson y las matemáticas. Madrid: Ariel.

(15) Socorro, F. (15 de septiembre de 2004). DeGerencia.com. Recuperado el 10 de junio de 2019, de Redes Sociales: Una aproximación al concepto: https://degerencia.com/articulo/ redes_sociales_una_aproximacion_al_concepto/

(16) Suárez-Iñiguez, E. (2004). ¿Son ciencias las sociales? Estudios Políticos, 13-25. 